Copyright (C 2016 IEEE. Personal use of this material is permitted. Permission from IEEE must be obtained for all other uses, in any current or future media, including reprinting/republishing this material for advertising or promotional purposes, creating new collective works, for resale or redistribution to servers or lists, or reuse of any copyrighted component of this work in other works. 


\title{
Globally monotonic tracking control of multivariable systems
}

\author{
Lorenzo Ntogramatzidis, Jean-François Trégouët, Robert Schmid and Augusto Ferrante
}

\begin{abstract}
In this paper we present a method for designing a linear time invariant (LTI) state-feedback controller to monotonically track a step reference at any desired rate of convergence for any initial condition. This method is developed for multi-input multioutput (MIMO) systems, and can be applied to strictly/nonstrictly proper systems, and also minimum/nonminimum-phase systems. This framework shows that for MIMO systems the objectives of achieving a rapid settling time, while at the same time avoiding overshoot/undershoot, are not always competing objectives.
\end{abstract}

\section{INTRODUCTION}

The problem of improving the shape of the step response curve for linear time invariant (LTI) systems is as old as control theory. Its relevance is seen in countless applications such as heating/cooling systems, elevator and satellite positioning, automobile cruise control and the positioning of a CD disk $\mathrm{read} / \mathrm{write}$ head. When dealing with the transient performance, one is usually concerned with the task of reducing both the overshoot and the undershoot, or, ideally, of achieving a monotonic response that rapidly converges to the steadystate value. It is commonly understood that the objectives of achieving a rapid settling time, while at the same time avoiding overshoot and undershoot, are competing objectives in the controller design, and must be dealt with by seeking a tradeoff, see e.g. [1]. While this is certainly the case for single-input single-output (SISO) systems, the control methods we develop in this paper challenge this widely-held perception for the MIMO case. We show that in the case of LTI MIMO systems, it is possible to achieve arbitrarily fast settling time and a monotonic step response in all outputs for any initial condition, which implies the avoidance of overshoot/undershoot, even in the presence of nonminimum-phase zeros.

In contrast with the extensive literature for SISO systems, which includes [2]-[8], to date there have been very few papers offering methods for avoiding undershoot or overshoot in the step response of MIMO systems, see e.g. [9]. The most famous among the classical methods that deal with tracking control problems is the model matching problem, see e.g. [10], [11].

A recent contribution offering design methods for MIMO systems is [12], where a procedure is proposed for the design of a state-feedback controller to yield a non-overshooting step

L. Ntogramatzidis is with the Department of Mathematics and Statistics, Curtin University, Perth, Australia. E-mail: L. Ntogramatzidisecurtin.edu.au.

J.-F. Trégouët is with Université de Lyon, Laboratoire Ampère CNRS UMR 5005, INSA-Lyon; F-69621, Villeurbanne, France. Email: jean-francois.tregouet@insa-lyon.fr.

R. Schmid is with the Department of Electrical and Electronic Engineering, The University of Melbourne, Parkville, VIC 3010, Australia. E-mail: rschmideunimelb.edu.au.

A. Ferrante is with the Dipartimento di Ingegneria dell'Informazione, Università di Padova, via Gradenigo, 6/B I-35131 Padova, Italy. E-mail: augusto@dei.unipd.it. response for LTI MIMO systems, by constraining the closedloop eigenstructure so that each component of the tracking error is driven only by a single real-valued closed-loop mode, which means that the output of the system is monotonic in each output component regardless of the initial condition, see also [13]. A key limitation of the methods in [12], [13] is the lack of conditions, given in terms of the system structure, that ensure the existence of a controller that delivers the desired transient response. Moreover, the design method involves a search for suitable closed-loop eigenvalues, and while this search can be conducted efficiently, the authors were unable to give conditions ensuring a satisfactory search outcome. The objective of this paper is to revisit the method of [12] to the end of developing conditions expressed in terms of the parameters of the system that guarantee that the design method will deliver a state-feedback controller that yields a monotonic step response from any initial condition and for any constant reference signal. When this goal is achievable, we say that the control yields a globally monotonic response, by which we mean that the same feedback matrix yields a monotonic response from all initial conditions, and with respect to all possible step references.

We also offer a parameterisation of all the feedback matrices that achieve global monotonicity, thus opening the door to the formulation of optimisation problems whose goal is to exploit the available freedom to address further objectives such as minimum gain or improved robustness of the closed-loop eigenstructure in the same spirit of [14]. For some less essential proofs, many examples and additional discussion we refer to a much longer and more detailed manuscript in [15].

Notation. The image and the kernel of matrix $A$ are denoted by $\operatorname{im} A$ and $\operatorname{ker} A$, respectively. The Moore-Penrose pseudoinverse of $A$ is denoted by $A^{\dagger}$. When $A$ is square, we denote by $\sigma(A)$ the spectrum of $A$. If $A: \mathscr{X} \longrightarrow \mathscr{Y}$ is a linear map and if $\mathscr{J} \subseteq \mathscr{X}$, the restriction of the map $A$ to $\mathscr{J}$ is denoted by $A \mid \mathscr{J}$. If $\mathscr{X}=\mathscr{Y}$ and $\mathscr{J}$ is $A$-invariant, the eigenstructure of $A$ restricted to $\mathscr{J}$ is denoted by $\sigma(A \mid \mathscr{J})$. If $\mathscr{J}_{1}$ and $\mathscr{J}_{2}$ are $A$-invariant subspaces and $\mathscr{J}_{1} \subseteq \mathscr{J}_{2}$, the mapping induced by $A$ on the quotient space $\mathscr{J}_{2} / \mathscr{J}_{1}$ is denoted by $A \mid \mathscr{J}_{2} / \mathscr{J}_{1}$, and its spectrum is denoted by $\sigma\left(A \mid \mathscr{J}_{2} / \mathscr{J}_{1}\right)$. The symbol $\oplus$ stands for the direct sum of subspaces. The symbol $\uplus$ denotes union with any common elements repeated. Given a map $A: \mathscr{X} \longrightarrow \mathscr{X}$ and a subspace $\mathscr{B}$ of $\mathscr{X}$, we denote by $\langle A, \mathscr{B}\rangle$ the smallest $A$-invariant subspace of $\mathscr{X}$ containing $\mathscr{B}$. Given a complex matrix $M$, the symbol $M^{*}$ denotes the conjugate transpose of $M$. Moreover, we denote by $M_{i}$ its $i$-th row and by $M^{j}$ its $j$-th column, respectively. Given a finite set $S$, the symbol $2^{S}$ denotes the power set of $S$, while card $(S)$ stands for the cardinality of $S$. Consider the set $\Phi=\left\{\pi \in \mathbb{R}^{n} \mid \Pi(\pi)=1\right\}$, where $\Pi: \mathbb{R}^{n} \longrightarrow\{0,1\}$. If $\Phi$ is nonempty and its complement is formed by the solutions to finitely many polynomial equa- 
tions $p_{i}\left(x_{1}, \ldots, x_{n}\right)=0(i=1, \ldots, k)$ where the coefficients of the $p_{i}$ are real, then we say that almost all $\pi \in \mathbb{R}^{n}$ satisfy $\Pi$.

\section{Problem Formulation}

Consider the continuous-time LTI system $\Sigma$ governed by

$$
\Sigma:\left\{\begin{array}{l}
\dot{x}(t)=A x(t)+B u(t), \quad x(0)=x_{0}, \\
y(t)=C x(t)+D u(t),
\end{array}\right.
$$

where, for all $t \in \mathbb{R}, x(t) \in \mathscr{X}=\mathbb{R}^{n}$ is the state, $u(t) \in \mathscr{U}=\mathbb{R}^{m}$ is the control input, $y(t) \in \mathscr{Y}=\mathbb{R}^{p}$ is the output, and $A, B$, $C$ and $D$ are appropriate dimensional constant matrices. We assume that all the columns of $\left[\begin{array}{l}B \\ D\end{array}\right]$ and all the rows of $\left[\begin{array}{ll}C & D\end{array}\right]$ are linearly independent. The Rosenbrock matrix is defined as

$$
P_{\Sigma}(\lambda) \stackrel{\text { def }}{=}\left[\begin{array}{cc}
A-\lambda I_{n} & B \\
C & D
\end{array}\right], \quad \lambda \in \mathbb{C} .
$$

The invariant zeros of $\Sigma$ are the values of $\lambda \in \mathbb{C}$ for which the rank of $P_{\Sigma}(\lambda)$ is strictly smaller than its normal rank. The set of zeros of $\Sigma$ is denoted by $\mathscr{Z}$, and the set of minimum-phase (lying in the left-hand complex plane) zeros of $\Sigma$ is $\mathscr{Z}_{g}$.

We denote by $\mathscr{V}^{\star}$ the largest output-nulling subspace of $\Sigma$, i.e., the largest subspace $\mathscr{V}$ of $\mathscr{X}$ for which a matrix $F \in \mathbb{R}^{m \times n}$ exists such that $(A+B F) \mathscr{V} \subseteq \mathscr{V} \subseteq \operatorname{ker}(C+D F)$. Any real matrix $F$ satisfying this inclusion is called a friend of $\mathscr{V}$. We denote by $\mathfrak{F}(\mathscr{V})$ the set of friends of $\mathscr{V}$. The symbol $\mathscr{R}^{\star}$ denotes the so-called output-nulling reachability subspace on $\mathscr{V}^{\star}$. The closed-loop spectrum can be partitioned as $\sigma(A+$ $B F)=\sigma\left(A+B F \mid \mathscr{V}^{\star}\right) \uplus \sigma\left(A+B F \mid \mathscr{X} / \mathscr{V}^{\star}\right)$. Further, we have $\sigma\left(A+B F \mid \mathscr{V}^{\star}\right)=\sigma\left(A+B F \mid \mathscr{R}^{\star}\right) \uplus \sigma\left(A+B F \mid \mathscr{V}^{\star} / \mathscr{R}^{\star}\right)$, where $\sigma\left(A+B F \mid \mathscr{R}^{\star}\right)$ is freely assignable with a suitable $F \in \mathfrak{F}\left(\mathscr{V}^{\star}\right)$, whereas $\sigma\left(A+B F \mid \mathscr{V}^{\star} / \mathscr{R}^{\star}\right)$ is fixed for every $F \in \mathfrak{F}\left(\mathscr{V}^{\star}\right)$ and coincide with the invariant zero structure of $\Sigma$, [16, Theorem 7.19]. Finally, the symbol $\mathscr{V}_{g}^{\star}$ denotes the largest stabilisability output-nulling subspace of $\Sigma$.

\section{A. The tracking control problem}

In this paper, we are concerned with the problem of the design of a state-feedback control law for (1) such that for all initial conditions the output $y$ tracks a step reference $r \in \mathscr{Y}$ with zero steady-state error and is monotonic in all components. The following assumption ensures that any target $r$ can be tracked from any $x_{0}$ :

Assumption 2.1: System $\Sigma$ is right invertible and stabilisable, and has no invariant zeros at the origin.

We also make the following assumption.

Assumption 2.2: $\Sigma$ has no coincident minimum-phase zeros.

This assumption does not lead to a significant loss of generality. In fact, the case of coincident zeros can be dealt with by using the procedure described in [17]. Both Assumptions 2.1-2.2 are standing assumptions in this paper.

Given the step reference $r \in \mathscr{Y}$ to track, let $F$ be such that $A+B F$ is asymptotically stable. Let $x_{\mathrm{ss}} \in \mathscr{X}$ and $u_{\mathrm{ss}} \in \mathscr{U}$ be such that, for the given $r \in \mathscr{Y}$, there hold

$$
0=A x_{\mathrm{ss}}+B u_{\mathrm{ss}}, \quad r=C x_{\mathrm{ss}}+D u_{\mathrm{ss}} .
$$

Applying the state-feedback control law

$$
u(t)=F\left(x(t)-x_{\mathrm{ss}}\right)+u_{\mathrm{ss}}
$$

to (1), changing variable $\xi \stackrel{\text { def }}{=} x-x_{\mathrm{ss}}$ and defining the tracking error $\varepsilon(t) \stackrel{\text { def }}{=} y(t)-r(t)$ gives the closed-loop system

$$
\Sigma_{\text {aut }}:\left\{\begin{array}{l}
\dot{\xi}(t)=(A+B F) \xi(t), \quad \xi(0)=\xi_{0}=x_{0}-x_{\mathrm{ss}}, \\
\varepsilon(t)=(C+D F) \xi(t) .
\end{array}\right.
$$

Since $A+B F$ is asymptotically stable, $x$ converges to $x_{\mathrm{ss}}, \xi$ converges to zero and $y$ converges to $r$ as $t$ goes to infinity.

In this paper we are concerned with the problem of finding a matrix $F$ such that the closed-loop system obtained using (4) achieves a monotonic response at any desired rate of convergence, from all initial conditions. We shall describe this property as global monotonicity.

Problem 1: Let $\rho<0$. Find $F$ that yields an asymptotically stable system $\Sigma_{\text {aut }}$ for which $\varepsilon(t)$ converges monotonically to 0 at an exponential rate of at least $\rho$ in all outputs from all $x_{0}$.

If we achieve a tracking error $\varepsilon(t)$ that has a single exponential per component and we can choose each $\lambda_{k}$ so that $\lambda_{k} \leq \rho$, then we solve Problem 1 , since exponentials of $\lambda_{k}$ are monotonic functions. Thus, a possible way of solving Problem 1 consists in the solution of the following problem.

Problem 2: Let $\rho<0$. Find $F$ that yields an asymptotically stable system $\Sigma_{a u t}$ for which $\varepsilon(t)$ is given, from all $x_{0}$, by

$$
\varepsilon(t)=\left[\begin{array}{llll}
\beta_{1} e^{\lambda_{1} t} & \beta_{2} e^{\lambda_{2} t} & \ldots & \beta_{p} e^{\lambda_{p} t}
\end{array}\right]^{\top}
$$

for some real coefficients $\left\{\beta_{k}\right\}_{k=1}^{p}$ depending only upon $\xi_{0}$ and for some real values $\lambda_{1}, \lambda_{2}, \cdots, \lambda_{p}$ satisfying $\lambda_{k} \leq \rho$.

Solutions of Problem 2 solve Problem 1. In [15, Lemma 1] it is shown that the converse is also true. In other words, Problem 1 is equivalent to Problem 2.

Another important and useful problem is one in which the requirements include a specified choice of the closed-loop modes that are visible in each component of the tracking error:

Problem 3: Let $\lambda_{1}, \cdots, \lambda_{p} \in \mathbb{R}^{-}$. Find $F$ that yields an asymptotically stable closed-loop system $\Sigma_{a u t}$ for which, from $x_{0}$ and $r$, the tracking error term $\varepsilon$ is given by (6).

Remark 1: An anonymous reviewer proposed the following solution to these problems in the case where $D$ is the zero matrix, which consists in decomposing the system as

$$
A=\left[\begin{array}{ll}
A_{11} & A_{12} \\
A_{21} & A_{22}
\end{array}\right], B=\left[\begin{array}{l}
B_{1} \\
B_{2}
\end{array}\right], C=\left[\begin{array}{ll}
0 & I_{p}
\end{array}\right],
$$

so that the problem is to guarantee by means of a state feedback $K=\left[\begin{array}{ll}K_{1} & K_{2}\end{array}\right]$ that $x_{2}=y$ has a monotonic behavior for all initial states. This problem is easily seen to be equivalent to the solvability of the following conditions: i) The equation $A_{21}+B_{2} K_{1}=0$ has a solution; if this is the case, we parameterize its solutions as $K_{1}=\bar{K}_{1}+H \tilde{K}_{1}$, where $H$ is a basis matrix of $\operatorname{ker} B_{2}$ and $\tilde{K}_{1}$ is arbitrary; ii) $A_{11}+B_{1} K_{1}$ is asymptotically stable, i.e., $\left(A_{11}+B_{1} \bar{K}_{1}, B_{1} T\right)$ is stabilizable; iii) $A_{22}+B_{2} K_{2}=\Lambda$ has a solution, where $\Lambda=\operatorname{diag}\left\{\lambda_{1}, \cdots, \lambda_{p}\right\}$. Hence, this solution, when $D=0$, characterizes the solvability of our problem since, using this feedback, the closed-loop matrix becomes $\left[\begin{array}{cc}A_{11}+B_{1} K_{1} & A_{12}+B_{1} K_{2} \\ 0 & \Lambda\end{array}\right]$. The rest of this paper is devoted to finding solvability conditions for this problem expressed in terms of the problem data. This will offer new insight into how the solution changes in terms of the variations of the problem data, and will allow us to recover well-known results on the impossibility of obtaining a monotonic response 
for SISO strictly proper systems with a real nonminimumphase zero.

\section{MATHEMATICAL BACKGROUND}

The first tool needed in this paper is $\mathscr{V}_{g}^{\star}$, which is made up of the sum of two parts. The first part is $\mathscr{R}^{\star}$. The second is the subspace spanned by the directions associated with the minimum-phase invariant zeros of $\Sigma$. We recall some important results concerning the relations between these subspaces and the null-space of the Rosenbrock matrix $P_{\Sigma}$. Given $\mu \in \mathbb{C}$, the symbol $N_{\Sigma}(\mu)$ denotes a basis matrix for $\operatorname{ker} P_{\Sigma}(\mu)$.

The following result, see [14], presents a procedure for the computation of a basis for $\mathscr{V}_{g}^{\star}$ and $\mathscr{R}^{\star}$ and for the parameterisation of all their friends that place the free eigenvalues of the closed-loop restricted to them at arbitrary locations.

The complex numbers $\mu_{1}, \ldots, \mu_{h}$ are said to be $s$-indexed if $2 s \leq h$ and $\mu_{1}, \ldots, \mu_{2 s}$ are in $\mathbb{C} \backslash \mathbb{R}$, while the remaining $\mu_{2 s+1}, \ldots, \mu_{h}$ are real, and for all odd $k \leq 2 s$ we have $\mu_{k+1}=$ $\mu_{k}^{*}$. For every odd $i \in\{1, \ldots, 2 s\}$, let the basis matrix $N_{\Sigma}\left(\mu_{i+1}\right)$ be constructed as $N_{\Sigma}\left(\mu_{i+1}\right)=N_{\Sigma}\left(\mu_{i}^{*}\right)=N_{\Sigma}\left(\mu_{i}\right)^{*}$.

Lemma 1: ([14]). Let $r=\operatorname{dim} \mathscr{R}^{\star}$. Let $z_{1}, \ldots, z_{t}$ be the $s_{z^{-}}$ indexed minimum-phase invariant zeros of $\Sigma$. Let $\mu_{1}, \ldots, \mu_{r} \in$ $\mathbb{R} \backslash \mathscr{Z}$ be $s$-indexed. Let also

$M_{\Sigma}\left(z_{k}\right) \stackrel{\text { def }}{=} \begin{cases}N_{\Sigma}\left(z_{k}\right)+N_{\Sigma}\left(z_{k+1}\right) & \text { if } k \in\left\{1, \ldots, 2 s_{z}\right\} \text { is odd } \\ i\left[N_{\Sigma}\left(z_{k}\right)-N_{\Sigma}\left(z_{k-1}\right)\right] & \text { if } k \in\left\{1, \ldots, 2 s_{z}\right\} \text { is even } \\ N_{\Sigma}\left(z_{k}\right) & \text { if } k \in\left\{2 s_{z}+1, \ldots, t\right\}\end{cases}$

and $\left[\begin{array}{c}\hat{V}_{g} \\ \hat{W}_{g}\end{array}\right] \stackrel{\text { def }}{=}\left[N_{\Sigma}\left(\mu_{1}\right)|\ldots| N_{\Sigma}\left(\mu_{r}\right)\left|M_{\Sigma}\left(z_{1}\right)\right| \ldots \mid M_{\Sigma}\left(z_{t}\right)\right]$. Then, the columns of $\hat{V}_{g}$ span $\mathscr{V}_{g}^{\star}$. Let $\ell_{k}$ and $\eta_{k}$ be the number of columns of $N_{\Sigma}\left(\mu_{k}\right)$ and $M_{\Sigma}\left(z_{k}\right)$, respectively. Then, the columns of $V_{K, H} \stackrel{\text { def }}{=} \hat{V}_{g} \operatorname{diag}\{K, H\}$, with $K \stackrel{\text { def }}{=} \operatorname{diag}\left\{k_{1}, \ldots, k_{r}\right\}$ and $H \stackrel{\text { def }}{=} \operatorname{diag}\left\{h_{1}, \ldots, h_{t}\right\}$ are a basis for $\mathscr{V}_{g}^{\star}$ adapted to $\mathscr{R}^{\star}$ for almost all $k_{i} \in \mathbb{R}^{\ell_{i}}$ and $h_{i} \in \mathbb{R}^{\eta_{i}}$. Finally, defining $W_{K, H} \stackrel{\text { def }}{=} \hat{W}_{g} \operatorname{diag}\{K, H\}$, the set of all friends $F$ of $\mathscr{V}_{g}^{\star}$ such that $\sigma\left(A+B F \mid \mathscr{V}_{g}^{\star}\right)=\left\{\mu_{1}, \ldots, \mu_{r}\right\} \uplus\left\{z_{1}, \ldots, z_{t}\right\}$ is parameterised in $K$ and $H$ as $F_{K, H}=W_{K, H} V_{K, H}^{\dagger}$, where $K$ and $H$ are such that $\operatorname{rank} V_{K, H}=\operatorname{dim} \mathscr{V}_{g}^{\star}$.

If in Lemma 1 we construct $\left[\begin{array}{c}\hat{V}_{g} \\ \hat{W}_{g}\end{array}\right]$ without $M_{\Sigma}\left(z_{i}\right)$, the columns of $V_{K} \stackrel{\text { def }}{=} \hat{V}_{g} K$, with $K \stackrel{\text { def }}{=} \operatorname{diag}\left\{k_{1}, \ldots, k_{r}\right\}$ are a basis for $\mathscr{R}^{\star}$ for almost all $k_{i} \in \mathbb{R}^{\ell_{i}}$, and defining $W_{K} \stackrel{\text { def }}{=} \hat{W}_{g} K$, the set of friends $F$ of $\mathscr{R}^{\star}$ such that $\sigma\left(A+B F \mid \mathscr{R}^{\star}\right)=\left\{\mu_{1}, \ldots, \mu_{r}\right\}$ is parameterised in $K$ as $F_{K}=W_{K} V_{K}^{\dagger}$, where $K$ is such that $\operatorname{rank} V_{K}=\operatorname{dim} \mathscr{R}^{\star}$. Thus, we can write a spanning set of $\mathscr{R}^{\star}$ as

$$
\mathscr{R}^{\star}=\mathscr{R}^{\star}\left(\mu_{1}\right)+\cdots+\mathscr{R}^{\star}\left(\mu_{r}\right),
$$

where $\mu_{1}, \cdots, \mu_{r} \in \mathbb{R} \backslash \mathscr{Z}$, and where

$$
\mathscr{R}^{\star}(\mu) \stackrel{\text { def }}{=}\left\{v \in \mathscr{X} \mid \exists w \in \mathscr{U}:\left[\begin{array}{rr}
A-\mu I_{n} & B \\
C & D
\end{array}\right]\left[\begin{array}{l}
v \\
w
\end{array}\right]=0\right\} .
$$

\section{Solution to Problem 3}

As explained above, achieving a globally monotonic step response is equivalent to finding $F$ that distributes (at most) $p$ modes into the tracking error with one mode per component. To achieve this goal, let $\lambda \in \mathbb{R}$, and define

$$
\begin{aligned}
\hat{\mathscr{R}}_{j}(\lambda) \stackrel{\text { def }}{=}\{v \in \mathscr{X} \mid \exists \beta \in \mathbb{R} \backslash\{0\}, \exists w \in \mathscr{U}: \\
{\left.\left[\begin{array}{rr}
A-\lambda I_{n} & B \\
C & D
\end{array}\right]\left[\begin{array}{l}
v \\
w
\end{array}\right]=\left[\begin{array}{c}
0 \\
\beta e_{j}
\end{array}\right]\right\}, }
\end{aligned}
$$

for al $j \in\{1, \ldots, p\}$. The set $\hat{\mathscr{R}}_{j}(\lambda)$ represents the set of initial states such that a feedback matrix $F$ exists that renders all the output components identically zero with the only exception of the $j$-th component, which must be non-zero. Given $v \in \hat{\mathscr{R}}_{j}(\lambda)$ with $v \neq 0$ and $\beta \in \mathbb{R} \backslash\{0\}$ and $w \in \mathscr{U}$ such that $\left[\begin{array}{rr}A-\lambda I_{n} & B \\ C & D\end{array}\right]\left[\begin{array}{c}v \\ w\end{array}\right]=\left[\begin{array}{c}0 \\ \beta e_{j}\end{array}\right]$, the feedback matrices satisfying $F v=w$ guarantee that for any $\xi_{0} \in \operatorname{span}\{v\}$ there hold $\varepsilon_{k} \equiv 0$ for all $k \in\{1, \ldots, p\} \backslash\{j\}$ and $\varepsilon_{j} \neq 0$.

The modes that are not distributed on the tracking error have to be made invisible from $\varepsilon(t)$. This goal can be achieved using $\mathscr{V}_{g}^{\star}$. Define $V_{K, H}$ and $W_{K, H}$ as in Lemma 1 and such that $h \stackrel{\text { def }}{=} \operatorname{dim} \mathscr{V}_{g}^{\star}=\operatorname{rank} V_{K, H}$. Let us partition these matrices as $V_{K, H}=\left[\begin{array}{llll}v_{g, 1} & v_{g, 2} & \ldots & v_{g, h}\end{array}\right]$ and $W_{K, H}=\left[\begin{array}{llll}w_{g, 1} & w_{g, 2} & \ldots & w_{g, h}\end{array}\right]$. Then, we have $\left[\begin{array}{rr}A-\mu_{i} I_{n} & B \\ C & D\end{array}\right]\left[\begin{array}{c}v_{g, i} \\ w_{g, i}\end{array}\right]=0$ since $K$ and $H$ are block diagonal (here we assume for the sake of simplicity that all the $\mu_{i}$ are real). It follows that there exists a feedback matrix $F$ satisfying $F V_{K, H}=W_{K, H}$ rendering all the output components identically zero for any initial states resulting in a linear combination of columns vectors of $V_{K, H}$. Thus, solving Problem 3 results in constructing a set of vectors $v_{1}, \ldots, v_{p}$ such that $v_{i} \in \hat{\mathscr{R}}_{j}(\lambda)$ which, together with the columns of $V_{K, H}$, spans $\mathscr{X}$.

Lemma 2 : Let $\lambda_{1}, \cdots, \lambda_{p} \in \mathbb{R}^{-}$. Problem 3 is solvable if and only if for all $j \in\{1, \ldots, p\}$ there exists $v_{j} \in \hat{\mathscr{R}}_{j}\left(\lambda_{j}\right)$ such that

$$
\mathscr{V}_{g}^{\star}+\operatorname{span}\left\{v_{1}, \cdots, v_{p}\right\}=\mathscr{X} .
$$

A proof of this result can be found in [15, Lemma 4]. Condition (10) says that:

(i) when $\operatorname{dim} \mathscr{V}_{g}^{\star}=n-p$, then $v_{1}, \ldots, v_{p}$ have to be linearly independent and they all must be independent from $\mathscr{V}_{g}^{\star}$. When this is the case, $\lambda_{1}, \ldots, \lambda_{p} \in \mathbb{R}^{-}$are all part of the closed-loop spectrum for any solution $F$ since the linear equation

$$
F_{K, H}\left[\begin{array}{llll}
V_{K, H} & v_{1} & \ldots & v_{p}
\end{array}\right]=\left[\begin{array}{llll}
W_{K, H} & w_{1} & \ldots & w_{p}
\end{array}\right] .
$$

is solvable, and any of its solutions $F_{K, H}$ ensures that $(A+$ $\left.B F_{K, H}\right) V_{K, H}=X_{g} V_{K, H}$ for a certain matrix $X_{g}$ such that $\sigma\left(X_{g}\right)=$ $\left\{\mu_{1}, \ldots, \mu_{h}\right\}$ and $\left(C+D F_{K, H}\right) V_{K, H}=0$, and that there exist $\beta_{i} \neq 0$ such that $\left[\begin{array}{cc}A-\lambda_{i} I_{n} & B \\ C & D\end{array}\right]\left[\begin{array}{c}v_{i} \\ w_{i}\end{array}\right]=\left[\begin{array}{c}0 \\ \beta_{i} e_{i}\end{array}\right]$, which gives $(A+$ $\left.B F_{K, H}\right) v_{i}=\lambda_{i} v_{i}$ and $\left(C+D F_{K, H}\right) v_{i}=\beta_{i} e_{i}$ for all $i \in\{1, \ldots, p\}$. Therefore, $\sigma\left(A+B F_{K, H}\right)=\left\{\lambda_{1}, \ldots, \lambda_{p}, \mu_{1}, \ldots, \mu_{n-p}\right\}$ and

$\varepsilon(t)=\left(C+D F_{K, H}\right) e^{\lambda_{1} t} v_{1} \gamma_{1}+\ldots+\left(C+D F_{K, H}\right) e^{\lambda_{p} t} v_{p} \gamma_{p}=\left[\begin{array}{c}\beta_{1} \gamma_{1} e^{\lambda_{1} t} \\ \vdots \\ \beta_{p} \gamma_{p} e^{\lambda_{p} t}\end{array}\right]$

for some $\gamma_{1}, \ldots, \gamma_{p} \in \mathbb{R} \backslash\{0\}$, as required.

(ii) when $\operatorname{dim} \mathscr{V}_{g}^{\star}>n-p$, it may be possible to exploit the excess in "good" dimension of $\mathscr{V}_{g}^{\star}$ to compensate for possibly dependent vector(s) $v_{i}$. In such a case, not only is the monotonic tracking control problem solvable, but we are able to also obtain a response that achieves instantaneous tracking in some outputs. If (10) is satisfied and $\operatorname{dim} \mathscr{V}_{g}^{\star}>n-p$, for any $v_{k}$ that is dependent on $\mathscr{V}_{g}^{\star}$ together with the remaining $v_{i}$, Problem 3 can be solved with a matrix $F$ such that $\lambda_{k}$ is not part of the closed-loop spectrum.

Corollary 1: If $\operatorname{dim} \mathscr{V}_{g}^{\star}<n-p$, then Problem 3 does not admit solutions. Moreover, if Problem 3 is solvable with a feedback matrix $F$ such that $\lambda_{i} \notin \sigma(A+B F)$ for some $i \in\{1, \ldots, p\}$, then $\operatorname{dim} \mathscr{V}_{g}^{\star}>n-p$. 
Remark 2: Whenever (10) is satisfied, Problem 3 can be solved with an arbitrary convergence rate. In fact, from the right invertibility of $(A, B, C, D)$, every uncontrollable eigenvalue is also an invariant zero of $\Sigma$. 1 Hence, every uncontrollable eigenvalue of the pair $(A, B)$ is rendered invisible at $\varepsilon(t)$, and it does not limit the rate of convergence.

Condition (10) is not easy to test, as the sets $\hat{\mathscr{R}}_{j}\left(\lambda_{j}\right)$ are not, in general, subspaces of $\mathscr{X}$. We now wish to write (10) in terms of subspaces which we now define. For each $j \in$ $\{1, \ldots, p\}$ let $\Sigma_{j} \stackrel{\text { def }}{=}\left(A, B, C_{(j)}, D_{(j)}\right)$, where $C_{(j)} \in \mathbb{R}^{(p-1) \times n}$ and $D_{(j)} \in \mathbb{R}^{(p-1) \times m}$ are obtained by removing the $j$-th row from $C$ and $D$, respectively. The right invertibility of $(A, B, C, D)$ guarantees that $\mathscr{Z}$ contains the set of zeros $\mathscr{Z}_{j}$ of $\Sigma_{j}$ for any $j \in\{1, \ldots, p\}$. The largest output nulling reachability subspace of $\Sigma_{j}$ is denoted by $\mathscr{R}_{j}^{\star}$. Similarly to what was done for $\mathscr{R}^{\star}$ in (7), for distinct $\mu_{1}, \cdots, \mu_{r_{j}} \in \mathbb{R} \backslash \mathscr{Z}$, we decompose $\mathscr{R}_{j}^{\star}$ as

$$
\mathscr{R}_{j}^{\star}=\mathscr{R}_{j}^{\star}\left(\mu_{1}\right)+\cdots+\mathscr{R}_{j}^{\star}\left(\mu_{r_{j}}\right),
$$

where $\mathscr{R}_{j}^{\star}(\mu) \stackrel{\text { def }}{=}\left\{v \in \mathscr{X} \mid \exists w \in \mathscr{U}:\left[\begin{array}{cc}A-\mu I_{n} & B \\ C_{(j)} & D_{(j)}\end{array}\right]\left[\begin{array}{c}v \\ w\end{array}\right]=0\right\}$ and $r_{j}=\operatorname{dim} \mathscr{R}_{j}^{\star}$.

Proposition 1: Let $\mu \in \mathbb{R} \backslash \mathscr{Z}$. For all $j \in\{1, \cdots, p\}$

$$
\mathscr{R}_{j}^{\star}(\mu)=\hat{\mathscr{R}}_{j}(\mu) \cup \mathscr{R}^{\star}(\mu) .
$$

Proof: We show that $\mathscr{R}_{j}^{\star}(\mu) \supseteq \hat{\mathscr{R}}_{j}(\mu) \cup \mathscr{R}^{\star}(\mu)$. We begin proving that $\hat{\mathscr{R}}_{j}(\mu) \subseteq \mathscr{R}_{j}^{\star}(\mu)$. Let $v \in \hat{\mathscr{R}}_{j}(\mu)$. There exist $w \in$ $\mathscr{U}$ and $\beta \in \mathbb{R} \backslash\{0\}$ such that $\left[\begin{array}{rr}A-\mu I_{n} & B \\ C & D\end{array}\right]\left[\begin{array}{l}v \\ w\end{array}\right]=\left[\begin{array}{c}0 \\ \beta e_{j}\end{array}\right]$, which gives $C_{(j)} v+D_{(j)} w=0$. Hence, $v \in \mathscr{R}_{j}^{\star}(\mu)$. We prove that $\mathscr{R}^{\star}(\mu) \subseteq \mathscr{R}_{j}^{\star}(\mu)$. Let $v \in \mathscr{R}^{\star}(\mu)$. Then, there exist $w \in \mathscr{U}$ such that $\left[\begin{array}{cc}A-\mu I_{n} & B \\ C & D\end{array}\right]\left[\begin{array}{l}v \\ w\end{array}\right]=\left[\begin{array}{l}0 \\ 0\end{array}\right]$, which again implies that $C_{(j)} v+$ $D_{(j)} w=0$, so that $v \in \mathscr{R}_{j}^{\star}(\mu)$. Hence, $\hat{\mathscr{R}}_{j}(\mu) \cup \mathscr{R}^{\star}(\mu) \subseteq$ $\mathscr{R}_{j}^{\star}(\mu)$ holds. We show that $\mathscr{R}_{j}^{\star}(\mu) \subseteq \hat{\mathscr{R}}_{j}(\mu) \cup \mathscr{R}^{\star}(\mu)$. Let $v \in \mathscr{R}_{j}^{\star}(\mu)$. There exists $w \in \mathscr{U}$ such that $\left[\begin{array}{cc}A-\mu I_{n} & B \\ C_{(j)} & D_{(j)}\end{array}\right]\left[\begin{array}{l}v \\ w\end{array}\right]=0$. Let $\beta=C_{j} v+D_{j} w$. Then, $\left[\begin{array}{cc}A-\mu I_{n} & B \\ C & D\end{array}\right]\left[\begin{array}{c}v \\ w\end{array}\right]=\left[\begin{array}{c}0 \\ \beta e_{j}\end{array}\right]$. If $\beta \neq 0$, we have $v \in \hat{\mathscr{R}}_{j}(\mu)$, whereas if $\beta=0$, we find $v \in \mathscr{R}^{\star}(\mu)$. Thus, $v \in \hat{\mathscr{R}}_{j}(\mu) \cup \mathscr{R}^{\star}(\mu)$.

Proposition 2: Let $\mu \in \mathbb{R} \backslash \mathscr{Z}$. For all $j \in\{1, \cdots, p\}, \mathscr{R}_{j}^{\star}(\mu)$ and $\hat{\mathscr{R}}_{j}(\mu)$ coincide almost everywhere.

Proof: Since $\Sigma$ is right invertible and $\mu$ is not a zero, the inclusion $\mathscr{R}^{\star}(\mu) \subseteq \mathscr{R}_{j}^{\star}(\mu)$ from Proposition 1 is strict. Indeed, in such a case, $\left[\begin{array}{ll}C_{j} & D_{j}\end{array}\right]$ is linearly independent from every row of $\left[\begin{array}{cc}A-\mu I_{n} & B \\ C_{(j)} & D_{(j)}\end{array}\right]$. Thus, $\operatorname{dim} \mathscr{R}^{\star}(\mu)<\operatorname{dim} \mathscr{R}_{j}^{\star}(\mu)$. Proposition 1 ensures that $\mathscr{R}_{j}^{\star}(\mu) \backslash \hat{\mathscr{R}}_{j}(\mu) \subseteq \mathscr{R}^{\star}(\mu)$, which in general does not hold as an equality since $\mathscr{R}^{\star}(\mu)$ and $\hat{\mathscr{R}}_{j}(\mu)$ may have non-zero intersection.

The fact that $\mathscr{R}_{j}^{\star}(\mu)$ is a subspace of $\mathscr{X}$ and it is coincident with $\hat{\mathscr{R}}_{j}(\mu)$ modulo a set of points belonging to a proper algebraic variety within $\mathscr{R}^{\star}(\mu)$ motivates its use, in preference to $\hat{\mathscr{R}}_{j}(\mu)$, to establish conditions for our tracking problem.

\footnotetext{
${ }^{1}$ Indeed, an uncontrollable eigenvalue $\lambda$ either belongs to $\sigma(A+$ $\left.B \Phi|\mathscr{X}| \mathscr{V}^{\star}+\langle A, \operatorname{im} B\rangle\right)$ or to $\sigma\left(A+B \Phi\left|\mathscr{V}^{\star}\right| \mathscr{R}^{\star}\right)$, where $\Phi \in \mathfrak{F}\left(\mathscr{V}^{\star}\right)$ Since $\langle A, \operatorname{im} B\rangle$ is contained in the smallest input-containing subspace $\mathscr{S}^{\star}$ of $\Sigma$ [16, Chapter 8], and right-invertibility is equivalent to $\mathscr{V}^{\star}+$ $\mathscr{S}^{\star}=\mathscr{X}$ since $[C D]$ is full row-rank [16, Theorem 8.27], then $\mathscr{V}^{\star}+$ $\langle A, \operatorname{im} B\rangle=\mathscr{X}$. Hence, $\lambda \in \sigma\left(A+B \Phi \mid \mathscr{V}^{\star} / \mathscr{R}^{\star}\right)=\mathscr{Z}$.
}

\section{A. Solution of Problem 3: The case $\operatorname{dim} \mathscr{V}_{g}^{\star}=n-p$}

We begin by presenting a famous result in combinatorics [18, Theorem 3] due to Radó.

Lemma 3: Let $P_{1}, \ldots, P_{s}$ be sets of a Euclidean space. There exists elements $p_{i} \in P_{i}$ for all $i \in\{1, \ldots, s\}$ such that $\left\{p_{1}, \ldots, p_{s}\right\}$ is a linearly independent set if and only if given $k$ numbers $v_{1}, \ldots, v_{k}$ such that $1 \leq v_{1}<v_{2}<\ldots<v_{k} \leq s$ for all $k \in\{1, \ldots, s\}$, the union $P_{v_{1}} \cup P_{V_{2}} \cup \ldots \cup P_{V_{k}}$ contains $k$ linearly independent elements.

Let us specialise this result for linear subspaces of $\mathbb{R}^{n}$.

Proposition 3: Let $\mathscr{P}_{1}, \ldots, \mathscr{P}_{s}$ be subspaces of $\mathscr{X}$. There exists elements $p_{i} \in \mathscr{P}_{i}$ for all $i \in\{1, \ldots, s\}$ such that $\left\{p_{1}, \ldots, p_{s}\right\}$ is linearly independent if and only if

$$
\operatorname{dim}\left(\sum_{j \in S} \mathscr{P}_{j}\right) \geq \operatorname{card}(S) \quad \forall S \in 2^{\{1, \ldots, s\}} .
$$

Proof: Let $k \in\{1, \ldots, s\}$ and $1 \leq v_{1}<\ldots<v_{k} \leq s$. Then, $\mathscr{P}_{v_{1}} \cup \ldots \cup \mathscr{P}_{v_{k}}$ contains $k$ linearly independent elements if and only if $\mathscr{P}_{v_{1}}+\ldots+\mathscr{P}_{v_{k}}$ contains $k$ linearly independent elements, which is equivalent to $\operatorname{dim}\left(\mathscr{P}_{v_{1}}+\ldots+\mathscr{P}_{v_{k}}\right) \geq k^{2}$

Corollary 2: Let $n$ be the dimension of the linear space $\mathscr{X}$. Let $\mathscr{P}_{g}, \mathscr{P}_{1}, \ldots, \mathscr{P}_{s}$ be subspaces of $\mathscr{X}$, and let $\operatorname{dim} \mathscr{P}_{g}=n-s$. There exists a linearly independent set $\left\{p_{g_{1}}, \ldots, p_{g_{n-s}}, p_{1}, \ldots, p_{s}\right\}$ such that $\operatorname{span}\left\{p_{g_{1}}, \ldots, p_{g_{n-s}}\right\} \subseteq \mathscr{P}_{g}$ and $p_{i} \in \mathscr{P}_{i}$ for all $i \in\{1, \ldots, s\}$ if and only if

$$
\operatorname{dim}\left(\mathscr{P}_{g}+\sum_{j \in S} \mathscr{P}_{j}\right) \geq(n-s)+\operatorname{card}(S) \quad \forall S \in 2^{\{1, \ldots, s\}} .
$$

Proof: Let $\mathscr{X}=\mathscr{X}_{1} \oplus \mathscr{X}_{2}$ where $\mathscr{X}_{1}=\mathscr{P}_{g}$. In these coordinates, a basis matrix for $\mathscr{P}_{g}$ is given by $\left[\begin{array}{c}I_{n-s} \\ 0_{s \times(n-s)}\end{array}\right]$. Denote by $\left[\begin{array}{l}P_{i, 1} \\ P_{i, 2}\end{array}\right]$ a basis matrix for $\mathscr{P}_{i}(i \in\{1, \ldots, s\})$, where $P_{i, 1}$ and $P_{i, 2}$ have $n-s$ and $s$ rows, respectively. We can find a linearly independent set $\left\{p_{g_{1}}, \ldots, p_{g_{n-s}}, p_{1}, \ldots, p_{s}\right\}$ such that $\operatorname{span}\left\{p_{g_{1}}, \ldots, p_{g_{n-s}}\right\} \subseteq \mathscr{P}_{g}$ and $p_{i} \in \mathscr{P}_{i}$ for all $i \in\{1, \ldots, s\}$ if and only if there exist $\tilde{p}_{1} \in \operatorname{im} P_{1,2}, \ldots, \tilde{p}_{s} \in \operatorname{im} P_{s, 2}$ such that $\left\{\tilde{p}_{1}, \ldots, \tilde{p}_{s}\right\}$ is linearly independent. In view of Proposition 3 this happens if and only if $\operatorname{dim}\left(\sum_{i \in S} \mathscr{P}_{v_{i}, 2}\right) \geq \operatorname{card}(S)$ for all $S \in 2^{\{1, \ldots, S\}}$ which readily gives (14).

Since in Lemma 2 it was shown that, when $\operatorname{dim} \mathscr{V}_{g}^{\star}=n-p$, Problem 3 is solvable if and only if there exist $v_{j} \in \hat{\mathscr{R}}_{j}\left(\lambda_{j}\right)$ (where $j \in\{1, \ldots, p\}$ ) satisfying $\mathscr{V}_{g}^{\star}+\operatorname{span}\left\{v_{1}, \cdots, v_{p}\right\}=\mathscr{X}$, and that in Proposition 2 it was shown that for any $\mu \in \mathbb{R} \backslash \mathscr{Z}$ the set $\hat{\mathscr{R}}_{j}(\mu)$ coincides with the subspace $\mathscr{R}_{j}^{\star}(\mu)$ modulo a set of points that are roots of an algebraic equation, Corollary 2 leads to the following result.

Theorem 1: Let $\operatorname{dim} \mathscr{V}_{g}^{\star}=n-p$. Let $\lambda_{1}, \cdots, \lambda_{p} \in \mathbb{R}^{-}$. Problem 3 is solvable if and only

$$
\operatorname{dim}\left(\mathscr{V}_{g}^{\star}+\sum_{j \in S} \mathscr{R}_{j}^{\star}\left(\lambda_{j}\right)\right) \geq(n-p)+\operatorname{card}(S) \quad \forall S \in 2^{\{1, \ldots, p\}} .
$$

So far we have shown that if condition (15) in Theorem 1 is satisfied, it is possible to find $v_{i} \in \hat{\mathscr{R}}_{i}\left(\lambda_{i}\right)$ for all $i \in\{1, \ldots, p\}$ such that $\operatorname{rank}\left[\begin{array}{llll}V_{g} & v_{1} & \ldots & v_{p}\end{array}\right]=n$, and this means that a feedback matrix with the desired properties exists. However, a

\footnotetext{
${ }^{2}$ Since $\mathscr{P}_{v_{1}}+\ldots+\mathscr{P}_{v_{k}} \supseteq \mathscr{P}_{v_{1}} \cup \ldots \cup \mathscr{P}_{v_{k}}$, if $\mathscr{P}_{v_{1}} \cup \ldots \cup \mathscr{P}_{v_{k}}$ contains at least $k$ linearly independent elements, then also $\mathscr{P}_{v_{1}}+$ $\ldots+\mathscr{P}_{v_{k}}$ contains $k$ linearly independent elements. The converse is also true because $\mathscr{P}_{v_{1}}+\ldots+\mathscr{P}_{v_{k}}$ is the span of $\mathscr{P}_{v_{1}} \cup \ldots \cup \mathscr{P}_{v_{k}}$.
} 
much stronger result holds: the vectors $v_{1}, \ldots, v_{p}$ can be chosen "almost randomly" from within $\mathscr{R}_{1}^{\star}\left(\lambda_{1}\right), \mathscr{R}_{2}^{\star}\left(\lambda_{2}\right), \ldots, \mathscr{R}_{p}^{\star}\left(\lambda_{p}\right)$, respectively, and the resulting feedback will almost certainly solve Problem 3 as the following result establishes.

Theorem 2: Let $\lambda_{1}, \ldots, \lambda_{p} \in \mathbb{R}^{-}$. Let $r=\operatorname{dim} \mathscr{R}^{\star}$ and $\operatorname{dim} \mathscr{V}_{g}^{\star}=n-p$. Let (15) hold. Let $\hat{V}_{g}$ and $\hat{W}_{g}$ be as in Lemma 1 for the stable complex numbers $\mu_{1}, \ldots, \mu_{r}$ and for the minimum-phase invariant zeros $z_{1}, \ldots, z_{t}$. Let $\left[\begin{array}{c}V_{i} \\ W_{i}\end{array}\right]$ denote a basis matrix for $\operatorname{ker} P_{\Sigma_{i}}\left(\lambda_{i}\right)=\left[\begin{array}{cc}A-\lambda_{i} I_{n} & B \\ C_{(i)} & D_{(i)}\end{array}\right]$ for all $i \in\{1, \ldots, p\}$, where each $V_{i}$ and $W_{i}$ have $n$ and $m$ rows, respectively. Let

$$
\begin{aligned}
V_{K, H, k_{1}, \ldots, k_{p}} \stackrel{\text { def }}{=}\left[\begin{array}{lllll}
\hat{V}_{g} \operatorname{diag}\{K, H\} & V_{1} k_{1} & V_{2} k_{2} & \ldots & V_{p} k_{p}
\end{array}\right], \\
W_{K, H, k_{1}, \ldots, k_{p}} \stackrel{\text { def }}{=}\left[\begin{array}{llllll}
\hat{W}_{g} \operatorname{diag}\{K, H\} & W_{1} k_{1} & W_{2} k_{2} & \ldots & W_{p} k_{p}
\end{array}\right]
\end{aligned}
$$

where $k_{1}, \ldots, k_{p} \neq 0$ are real parameter vectors of appropriate sizes and $K$ and $H$ are block diagonal parameter matrices as in Lemma 1 such that $\operatorname{im}\left(\hat{V}_{g} \operatorname{diag}\{K, H\}\right)=\mathscr{V}_{g}^{\star}$. Then (1) $\operatorname{rank} V_{K, H, k_{1}, \ldots, k_{p}}=n$ for almost all $K$ and $H$ and $k_{1}, \ldots, k_{p} \neq 0$ as constructed above; (2) The set of all feedback matrices that solve Problem 3 for the given $\mu_{1}, \ldots, \mu_{r}$ is given by

$$
F_{K, H, k_{1}, \ldots, k_{p}}=W_{K, H, k_{1}, \ldots, k_{p}} V_{K, H, k_{1}, \ldots, k_{p}}^{-1} .
$$

Proof: In view of Theorem 1 and Lemma 2, there exist $v_{j} \in$ $\hat{\mathscr{R}}_{j}\left(\lambda_{j}\right)$ such that $(10)$ holds. Since $\hat{\mathscr{R}}_{j}\left(\lambda_{j}\right) \subseteq \mathscr{R}_{j}^{\star}\left(\lambda_{j}\right)$, there exist real vectors $k_{1}, \ldots, k_{p}$ such that $\operatorname{rank} \mathfrak{A}=n$, where

$\mathfrak{A} \stackrel{\text { def }}{=}\left[\hat{V}_{g} \operatorname{diag}\{K, H\}\left|V_{1}\right| \ldots \mid V_{p}\right] \operatorname{diag}\left\{I_{n-p}, k_{1}, \ldots, k_{p}\right\}$.

Since $\operatorname{rank}\left[\hat{V}_{g} \operatorname{diag}\{K, H\}\left|V_{1}\right| \ldots \mid V_{p}\right]=n$ in view of (15) written for $S=\{1, \ldots, p\}$, we conclude that $\mathfrak{A}$ loses rank only for $k_{1}, \ldots, k_{p}$ that solve a finite set of linear equations.

It remains to show that the parameterisation (16) of the feedback matrices which solve Problem 3 is exhaustive, i.e., that given a feedback $F$ which solves Problem 3 for $\lambda_{1}, \ldots, \lambda_{p}$, there exist $H, K, k_{1}, \ldots, k_{p}$ such that, computing $V_{K, H, k_{1}, \ldots, k_{p}}$ and $W_{K, H, k_{1}, \ldots, k_{p}}$ as in the statement, $F$ can be written as $W_{K, H, k_{1}, \ldots, k_{p}} V_{K, H, k_{1}, \ldots, k_{p}}^{-1}$. In view of Lemma $2, F$ satisfies

$\left[\begin{array}{c}A+B F \\ C+D F\end{array}\right] V_{g}=\left[\begin{array}{c}V_{g} \\ 0\end{array}\right] X_{g}, \quad\left[\begin{array}{c}A+B F \\ C_{(i)}+D_{(i)} F\end{array}\right] v_{i}=\left[\begin{array}{c}v_{i} \\ 0\end{array}\right] \lambda_{i}$,

where $V_{g}$ is a basis matrix for $\mathscr{V}_{g}^{\star}$, where $X_{g}$ is asymptotically stable, and with $i \in\{1, \ldots, p\}$, where $v_{i} \in \hat{\mathscr{R}}_{i}\left(\lambda_{i}\right)$. Assuming for simplicity that all the eigenvalues of $X_{g}$ are real and distinct, ${ }^{3}$ we can find a change of basis $T$ in $\mathscr{X}$ such that $X_{\triangle} \stackrel{\text { def }}{=} T^{-1} X_{g} T$ is diagonal. Denoting by $v_{i}$ the $i$-th column of $V_{g} T$, and by $\left\{\mu_{1}, \ldots, \mu_{n-p}\right\}$ the eigenvalues of $X_{\triangle}$, the first in (18) yields

$$
\left[\begin{array}{l}
A+B F \\
C+D F
\end{array}\right]\left[\begin{array}{lll}
v_{1} & \ldots & v_{n-p}
\end{array}\right]=\left[\begin{array}{ccc}
v_{1} & \ldots & v_{n-p} \\
0 & \ldots & 0
\end{array}\right]\left[\begin{array}{ccc}
\mu_{\eta(1)} & & \\
& \ddots & \mu_{\eta(n-p)}
\end{array}\right]
$$

where $\eta:\{1, \ldots, n-p\} \longrightarrow\{1, \ldots, n-p\}$ is a bijection. Defining $\omega_{i} \stackrel{\text { def }}{=} F v_{i}$, we find that $\left[\begin{array}{l}v_{i} \\ \omega_{i}\end{array}\right] \in \operatorname{ker}\left[\begin{array}{rr}A-\mu_{\eta_{i}} I_{n} & B \\ C & D\end{array}\right]$. We can repeat the same argument for the second in (18) (without the diagonalisation), and defining $w_{i}=F v_{i}$, there holds $\left[\begin{array}{c}v_{i} \\ w_{i}\end{array}\right] \in \operatorname{ker}\left[\begin{array}{cc}A-\lambda_{i} I_{n} & B \\ C_{(i)} & D_{(i)}\end{array}\right]$. Thus, (i) $F$ satisfies $\left[\omega_{1} \ldots \omega_{n-p} w_{1} \ldots w_{p}\right]=F\left[v_{1} \ldots v_{n-p} v_{1} \ldots v_{p}\right]$;

${ }^{3}$ The case of complex eigenvalues of $X_{g}$ can be dealt with using the argument in [14, Theorem 3.1]. (ii) $\quad\left[\begin{array}{llllll}\omega_{1} & \ldots & \omega_{n-p} & w_{1} & \ldots & w_{p}\end{array}\right]$ can be written as $W_{K, H, k_{1}, \ldots, k_{p}}$ for a suitable choice of $K, H$ and $k_{i}$; (iii) $\left[\begin{array}{lllllll}v_{1} & v_{2} & \ldots & v_{n-p} & v_{1} & \ldots & v_{p}\end{array}\right]$ can be written as $V_{K, H, k_{1}, \ldots, k_{p}}$ for suitable values of $K, H$ and $k_{i}$. Thus, $W_{K, H, k_{1}, \ldots, k_{p}}=F V_{K, H, k_{1}, \ldots, k_{p}}$.

\section{B. Solution of Problem 3: the general case}

We now consider the case where $h=\operatorname{dim} \mathscr{V}_{g}^{\star} \geq n-p$. The following generalisation of Radó's Theorem, see [19, Theorem 1.3], is the key to obtaining a necessary and sufficient solvability condition for Problem 3 in this general case.

Proposition 4: Let $\mathscr{P}_{1}, \ldots, \mathscr{P}_{s}$ be subspaces of $\mathscr{X}$. There exists $k$ elements $p_{1} \in \mathscr{P}_{i_{1}}, p_{2} \in \mathscr{P}_{i_{2}}, \ldots, p_{k} \in \mathscr{P}_{i_{k}}$ for some $1 \leq i_{1}<\ldots<i_{k} \leq s$ such that $\left\{p_{1}, \ldots, p_{k}\right\}$ is linearly independent if and only if

$$
\operatorname{dim}\left(\sum_{i \in S} \mathscr{P}_{i}\right) \geq \operatorname{card}(S)-(s-k)
$$

holds for all $S \in\left\{\mathfrak{S} \in 2^{\{1, \ldots, s\}} \mid \operatorname{card} \mathfrak{S}>s-k\right\}$.

As a result of Proposition 4, following the same argument of the proof of Corollary 2, one easily sees that a necessary and sufficient condition for Problem 3 is given as follows.

Theorem 3: Let $\lambda_{1}, \cdots, \lambda_{p} \in \mathbb{R}^{-}$. Problem 3 is solvable if and only if

$$
\begin{aligned}
\operatorname{dim}\left(\mathscr{V}_{g}^{\star}+\sum_{j \in S} \mathscr{R}_{j}^{\star}\left(\lambda_{j}\right)\right) \geq n-p+\operatorname{card}(\mathrm{S}) \\
\quad \forall S \in\left\{\mathfrak{S} \in 2^{\{1, \ldots, p\}} \mid \operatorname{card} \mathfrak{S}>h-(n-p)\right\} .
\end{aligned}
$$

It is clear that (21) reduces to (15) when $h=\operatorname{dim} \mathscr{V}_{g}^{\star}=$ $n-p$. The calculation of the feedback matrix does not change significantly with respect to the one outlined in Theorem 2 for the case $\operatorname{dim} \mathscr{V}_{g}^{\star}=n-p$. The main difference is that the $n \times(h+p)$ matrix $\mathfrak{V} \stackrel{\text { def }}{=}\left[\begin{array}{llll}\hat{V}_{g} \operatorname{diag}\{K, H\} & V_{1} k_{1} & \ldots & V_{p} k_{p}\end{array}\right]$ is not full column-rank. On the other hand, the rank of $\mathfrak{V}$ is $n$ for suitable values of the parameter matrices, which means that it is sufficient to eliminate from $\mathfrak{V}$ exactly $h+p-n$ columns that are linearly dependent upon the remaining $n$ columns.

We eliminate the corresponding columns of $\left[\begin{array}{llll}\hat{V}_{g} \operatorname{diag}\{K, H\} & V_{1} & \ldots & V_{p}\end{array}\right]$ and $\left[\hat{W}_{g} \operatorname{diag}\{K, H\} \quad W_{1} \ldots W_{p}\right]$, and we also eliminate the corresponding columns and rows from the parameter matrix $\operatorname{diag}\left\{I_{h}, k_{1}, k_{2}, \ldots, k_{p}\right\}$. We denote the matrices thus obtained by $\tilde{V}_{K, H, k_{1}, \ldots, k_{p}, \psi}, \tilde{W}_{K, H, k_{1}, \ldots, k_{p}, \psi}$ and $\tilde{K}_{K, H, k_{1}, \ldots, k_{p}, \psi}$ respectively, where $\psi$ is a mapping that represents the choice of the columns that have been eliminated. Now, the argument of Theorem 2 can be applied to the equation

$$
F_{K, H, k_{1}, \ldots, k_{p}, \psi} \tilde{V}_{K, H, k_{1}, \ldots, k_{p}, \psi}=\tilde{W}_{K, H, k_{1}, \ldots, k_{p}, \psi},
$$

which gives the solution to Problem 3 in parameterised form. We have just proved the following result.

Theorem 4: Let $\lambda_{1}, \ldots, \lambda_{p} \in \mathbb{R}^{-}$. Let $r=\operatorname{dim} \mathscr{R}^{\star}$, and let $h=\operatorname{dim} \mathscr{V}_{g}^{\star} \geq n-p$. Let (21) hold. Let $\hat{V}_{g}$ and $\hat{W}_{g}$ be constructed as in Lemma 1 for the asymptotically stable complex numbers $\mu_{1}, \ldots, \mu_{r}$ and for the minimum-phase invariant zeros $z_{1}, \ldots, z_{t}$. Let $\left[\begin{array}{c}V_{i} \\ W_{i}\end{array}\right]$ denote a basis for $\operatorname{ker} P_{\Sigma_{i}}\left(\lambda_{i}\right)=\left[\begin{array}{cc}A-\lambda_{i} I_{n} & B \\ C_{(i)} & D_{(i)}\end{array}\right]$ for all $i \in\{1, \ldots, p\}$. Let $v_{i}$ denote the number of columns of $V_{i}$. Let $k_{i} \in \mathbb{R}^{v_{i}}$ for all $i \in\{1, \ldots, p\}$. Let $\psi_{1}, \ldots, \psi_{n}$ be indexes of the columns of $V=\left[\begin{array}{llll}\hat{V}_{g} \operatorname{diag}\{K, H\} & V_{1} & \ldots & V_{p}\end{array}\right]$ such that the rank of $\tilde{V}_{K, H, k_{1}, \ldots, k_{p}, \psi}=\left[\begin{array}{lll}V^{\psi_{1}} & \ldots & V^{\psi_{n}}\end{array}\right]$ is $n$. Let 
$\tilde{W}_{K, H, k_{1}, \ldots, k_{p}, \psi}=\left[\begin{array}{lll}W^{\psi_{1}} & \ldots & W^{\psi_{n}}\end{array}\right]$, and let $\tilde{K}_{K, H, k_{1}, \ldots, k_{p}, \psi}$ be obtained from $\operatorname{diag}\left\{I_{h}, k_{1}, \ldots, k_{p}\right\}$ by removing the corresponding rows and columns. Then: (1) $\operatorname{rank} \tilde{V}_{K, H, k_{1}, \ldots, k_{p}, \psi}=n$ for almost all $K$ and $H$ as defined in Lemma 1 , for all $k_{1}, \ldots, k_{p} \neq 0$, and for all the choices $\psi$ such that the matrix obtained by eliminating $h+p-n$ columns from $V$ gives a matrix of rank $n$; (2) The set of feedback matrices that solve Problem 3 with $\mu_{1}, \ldots, \mu_{r}$, $z_{1}, \ldots, z_{t}$ and the given choice of $\psi$ is

$$
F_{K, H, k_{1}, \ldots, k_{p}, \psi}=\tilde{W}_{K, H, k_{1}, \ldots, k_{p}, \psi} \tilde{V}_{K, H, k_{1}, \ldots, k_{p}, \psi}^{-1},
$$

where $k_{i} \in \mathbb{R}^{v_{i}}, K$ and $H$ are block-diagonal matrices constructed as in Lemma 1 such that $\operatorname{im}\left(\hat{V}_{g} \operatorname{diag}\{K, H\}\right)=\mathscr{V}_{g}^{\star}$, and $\psi$ is such that the matrix obtained by eliminating $h+p-n$ columns from $V$ gives a matrix of rank $n$.

Remark 3: If $h=\operatorname{dim} \mathscr{V}_{g}^{\star}>n-p$, the natural choice is to build a feedback using a basis of $\mathscr{X}$ that uses as many basis vectors as possible from $\mathscr{V}_{g}^{\star}$, because every extra basis vector beyond the first $n-p$ potentially results in a tracking error with a zero component. This corresponds to selecting a mapping $\psi$ which eliminates as many columns $V_{i} k_{i}$ from $\mathfrak{V}$ as possible.

\section{Solution to Problem 1}

In this section, the solution to Problem 1 is investigated.

Theorem 5: Let $h \stackrel{\text { def }}{=} \operatorname{dim} \mathscr{V}_{g}^{\star}$. Problem 1 is solvable if and only if

$$
\operatorname{dim}\left(\mathscr{V}_{g}^{\star}+\sum_{j \in S} \mathscr{R}_{j}^{\star}\right) \geq n-p+\operatorname{card}(\mathrm{S})
$$

holds for all $S \in\left\{\mathfrak{S} \in 2^{\{1, \ldots, p\}} \mid \operatorname{card} \mathfrak{S}>h-(n-p)\right\}$.

Proof: Let for simplicity $h=n-p$. If (23) is not satisfied, there exists $S \in 2^{\{1, \ldots, p\}}$ such that $\operatorname{dim}\left(\mathscr{V}_{g}^{\star}+\sum_{j \in S} \mathscr{R}_{j}^{\star}\right)<n-$ $p+\operatorname{card}(\mathrm{S})$, which gives $\operatorname{dim}\left(\mathscr{V}_{g}^{\star}+\sum_{j \in S} \mathscr{R}_{j}^{\star}\left(\lambda_{j}\right)\right)<n-p+$ $\operatorname{card}(\mathrm{S})$ for any $\lambda_{1}, \cdots, \lambda_{p} \in \mathbb{R}^{-}$, since by (12) $\mathscr{R}_{j}^{\star}\left(\lambda_{j}\right) \subseteq \mathscr{R}_{j}^{\star}+$ $\mathscr{V}_{g}^{\star}$ for all $j \in\{1, \cdots, p\}$ and $\lambda_{j} \in \mathbb{R}$. In view of Theorem 1 , Problem 3 is never solvable, which implies that Problem 1 does not admit solution. Sufficiency follows from Corollary 2 .

We now consider the computation of the feedback matrix.

Theorem 6: Let $\operatorname{dim} \mathscr{V}_{g}^{\star}=n-p$. Let the condition in Theorem 5 hold. Let $\hat{V}_{g}$ and $\hat{W}_{g}$ be constructed as in Lemma 1. Let $\left[\begin{array}{c}V_{i}\left(\lambda_{i}\right) \\ W_{i}\left(\lambda_{i}\right)\end{array}\right]$ denote a polynomial basis matrix of least degree for the kernel of $P_{\Sigma_{i}}\left(\lambda_{i}\right)=\left[\begin{array}{cc}A-\lambda_{i} I_{n} & B \\ C_{(i)} & D_{(i)}\end{array}\right]$ for all $i \in\{1, \ldots, p\}$, where each $V_{i}\left(\lambda_{i}\right)$ and $W_{i}\left(\lambda_{i}\right)$ have $n$ and $m$ rows, respectively. Finally, let $v_{i}$ denote the number of columns of $V_{i}\left(\lambda_{i}\right)$. Let $k_{i} \in \mathbb{R}^{v_{i}}$ denote a parameter vector for all $i \in\{1, \ldots, p\}$. Let

$V_{K, H, k_{1}, \ldots, k_{p}}\left(\lambda_{1}, \ldots, \lambda_{p}\right) \stackrel{\text { def }}{=}\left[\hat{V}_{g} \operatorname{diag}\{K, H\} V_{1}\left(\lambda_{1}\right) k_{1} \ldots V_{p}\left(\lambda_{p}\right) k_{p}\right]$

$W_{K, H, k_{1}, \ldots, k_{p}}\left(\lambda_{1}, \ldots, \lambda_{p}\right) \stackrel{\text { def }}{=}\left[\hat{W}_{g} \operatorname{diag}\{K, H\} W_{1}\left(\lambda_{1}\right) k_{1} \ldots W_{p}\left(\lambda_{p}\right) k_{p}\right]$

Then: (1) $\operatorname{rank} V_{K, H, k_{1}, \ldots, k_{p}}\left(\lambda_{1}, \ldots, \lambda_{p}\right)=n$ for almost all $\lambda_{i} \in \mathbb{R}$, for almost all diagonal $K$ and $H$ as in Lemma 1 and for all $k_{i} \in \mathbb{R}^{v_{i}} \backslash\{0\}(i \in\{1, \ldots, p\})$; (2) The feedback matrices

$F_{K, H}\left(\lambda_{1}, \ldots, \lambda_{p}\right)=W_{K, H, k_{1}, \ldots, k_{p}}\left(\lambda_{1}, \ldots, \lambda_{p}\right) V_{K, H, k_{1}, \ldots, k_{p}}^{-1}\left(\lambda_{1}, \ldots, \lambda_{p}\right)$

obtained with $k_{i} \in \mathbb{R}^{v_{i}}$ and $\lambda_{i} \in \mathbb{R}^{-}(i \in\{1, \ldots, p\})$ such that $\operatorname{rank} V_{K, H, k_{1}, \ldots, k_{p}}\left(\lambda_{1}, \ldots, \lambda_{p}\right)=n$ are a solution to Problem 1 .

The proof follows essentially the steps of that of Theorem 4.

Remark 4: It is well known that a SISO strictly proper system with real nonminimum-phase zeros cannot be monotonic as undershoot must occur, [8]. This also follows as a particular case of Theorem 5. Indeed, the condition $\operatorname{dim} \mathscr{V}_{g}^{\star} \geq n-p$ follows from (23) when $S=\varnothing$, which is never satisfied for SISO strictly proper nonminimum-phase systems. In fact, $\operatorname{dim} \mathscr{V}_{g}^{\star} \leq \operatorname{dim} \mathscr{V}^{\star} \leq \operatorname{dim}(\operatorname{ker} C)=n-1$, where the first inequality can be an equality only if the system is minimum-phase.

\section{CONCLUDING REMARKS}

The problem of achieving a monotonic step response from any initial condition has been addressed for LTI MIMO systems. The results presented in this paper can be easily adapted to discrete-time systems as shown in [15]. Several numerical examples of the proposed method can also be found in [15].

\section{REFERENCES}

[1] G.F. Franklin, J.D. Powell, and A. Emami-Naeini, Feedback Control of Dynamic Systems, Addison-Wesley, Reading, MA, 3rd edition, 1994.

[2] J.B. Hoagg and D.S. Bernstein, Nonminimum-Phase Zeros, IEEE Control Systems Magazine, pp. 45-57, 2007.

[3] S.K. Lin and C.J. Fang, "Nonovershooting and monotone nondecreasing step responses of a third-order SISO linear system" IEEE Transactions on Automatic Control, 42: 1299-1303, 1997.

[4] J. Stewart and D.E. Davison, "On overshoot and nonminimum phase zeros". IEEE Transactions on Automatic Control, 51(8): 1378-1382, 2006.

[5] S. Darbha, and S.P. Bhattacharyya, "On the synthesis of controllers for a non-overshooting step response", IEEE Transactions on Automatic Control, 48(5): 797-799, 2003.

[6] M. Bement and S. Jayasuriya, "Use of state feedback to achieve a non-overshooting step response for a class of non-minimum phase systems", Journal of Dynamical Systems, Measurement and Control, 126(3): 657-660, 2004.

[7] S. Darbha, "On the synthesis of controllers for continuous time LTI systems that achieve a non-negative impulse response", Automatica, 39(1):159-165, 2003.

[8] R.H. Middleton, Trade-offs in linear control system design, Automatica, 27(2): 281-292, 1991.

[9] K. H. Johansson, Interaction bounds in multivariable control systems, Automatica, 38(6): 1045-1051, 2002.

[10] M. Malabre and V. Kučera, "Infinite structure and exact model matching problem: a geometric approach", IEEE Transactions on Automatic Control, 29(3): 266-268, 1984.

[11] V. Kučera, Analysis and Design of Discrete Linear Control Systems, Prentice-Hall, London, 1991.

[12] R. Schmid, and L. Ntogramatzidis, A unified method for the design of non-overshooting linear multivariable state-feedback tracking controllers. Automatica, 46(2): 312-321, 2010.

[13] R. Schmid, and L. Ntogramatzidis, The design of nonovershooting and non-undershooting multivariable state feedback tracking controllers. Systems \& Control Letters, 61(6): 714-722, 2012.

[14] L. Ntogramatzidis and R. Schmid, Robust eigenstructure assignment in geometric control theory. SIAM Journal of Control and Optimization. 52(2) : 960-986, 2014.

[15] L. Ntogramatzidis, J.-F. Trégouët, R. Schmid and A. Ferrante, A structural solution to the monotonic tracking control problem. http://arxiv.org/pdf/1412.1868v1.pdf.

[16] H. Trentelman, A. Stoorvogel, and M. Hautus, Control theory for linear systems, ser. Communications and Control Engineering. Great Britain: Springer, 2001.

[17] L. Ntogramatzidis, "Repeated eigenstructure assignment in the computation of friends of output-nulling subspaces". In Proc. $21^{\text {th }}$ Int. Symp. Math. Theory Networks and Syst. (MTNS 14), Groningen, The Netherlands, July 7-11, 2014.

[18] R. Radó, A theorem on independence relations. The Quarterly Journal of Mathematics, 13(1): 83-89, 1942.

[19] L. Mirsky and H. Perfect, Applications of the notion of independence to problems of combinatorial analysis. Journal of Combinatorial Theory, 2(3): 327-357, 1967. 